This work is licensed under a Creative Commons Attribution 4.0 International License https://creativecommons.org/licenses/by/4.0/

\title{
PRAGMATIC MARKER I PRAY IN EARLY MODERN ENGLISH
}

\author{
Natalia Yu. Merkuryeva \\ Moscow Metropolitan Governance Yury Luzhkov University, \\ 28, Sretenka st., Moscow, 107045, Russia
}

\begin{abstract}
The article deals with the sentences that contain pragmatic marker I pray you / thee and its variants as a parenthetical clause. The linguistic material for synchronic analysis is taken from the texts of the plays written by the English authors during the 16-1 $17^{\text {th }}$ centuries. The examination of the lexicalgrammatical and stylistic characteristics of the structures under analysis shows that the greatest variety of the marker forms is achieved at the beginning of the period under consideration. The following processes are seen to be the sources of the diversity: the increase of expression by means of an adverb (now, still, hartyly) or a modal verb, the change of the pronunciation of the constituent words followed by modifications of their representation in the text (comes into I prythee with variants prithee, prythee), the elimination of subject and / or object (resulting in the forms pray, pray you, I pray). Towards the end of the period under consideration, in the second half of the $17^{\text {th }}$ century, the diversity of the variants of the marker decreases, predominantly the following structures continue to exist: I pray, pray and I prythee. The parenthetical clause is studied in diachrony as well. The forms of the marker constituents, the peculiarities of the marker word order, the position of the clause in the sentences, the combination with other pragmatic markers are compared with the sentences from the texts of the $14-15^{\text {th }}$ centuries.
\end{abstract}

Key Words: pragmatic markers, discourse markers, historical syntax, historical pragmatics, authorial style, Early Modern English

For citation: Merkuryeva N.Yu. (2021). Pragmatic Marker I Pray in Early Modern English. Philology at MGIMO, 7(3), pp. 34-42. https://doi.org/10.24833/2410-2423-2021-3-27-34-42

\section{ПРАГМАТИЧЕСКИЙ МАРКЕР I РRAY В РАННЕНОВОАНГЛИЙСКИЙ ПЕРИОД}

\author{
Н.Ю. Меркурьева
}

Московский городской университет управления Правительства Москвы имени Ю.М. Лужкова, 107045, Россия, Москва, ул. Сретенка, 28 
Аннотация. Статья посвящена рассмотрению предложений, содержащих предикативный прагматический маркер-оборот I рrау уои / theе и его разновидности в качестве вводной клауземы. Лингвистический материал для синхронического изучения подобран из пьес английских авторов, созданных в XVI-XVII вв. Анализ лексико-грамматических и стилистических характеристик найденных структур показывает, что наибольшее разнообразие форм оборота достигается в начале исследуемого периода, в произведениях XVI в. Источниками разнообразия представляются несколько прочессов: увеличение объёма оборота за счёт добавления наречия (now, still, hartyly) или модального глагола, изменение в произношении составляющих оборот слов с одновременным изменением их отражения в тексте (оформившееся в разновидность I prytheе с вариантами prithee, pr'ytheе), элиминирование подлежащего и/или дополнения (с образованием борм рray, рray уои, I ргау). Ближе к кониу рассматриваемого периода, во второй половине XVII в., разнообразие разновидностей оборота значительно уменьшается, преимущественно сохраняющиеся структуры - I pray, pray и (I) prytheе. Производится исследование вводного предложения I pray уои / theе в диахронии (XIV-XVII вв.). Обсуждаются формы представления составляющих оборота, особенности словорасположения в нём, размещение оборота в конструкиии, комбинирование с другими прагматическими маркерами.

Ключевые слова: прагматические маркерь, дискурсивные маркерь, историческая прагматика, исторический синтаксис, авторский стиль, ранненовоанглийский период

Для цитирования: Меркурьева Н.Ю. (2021). Прагматический маркер I Pray в ранненовоанглийский период. Филологические науки в МГИМО. 7(3), С. 34-42. https://doi.org/10.24833/24102423-2021-3-27-34-42

\section{Введение.}

$\mathrm{P}$ анненовоанглийский период истории английского языка продолжался с XVI до первой половины XVIII в. [1, с. 301] и представляет интерес для лингвиста-историка, поскольку, как считается, в это время особенно интенсивно происходило и к концу периода завершилось формирование общенационального литературного стандарта, выполнявшего функции наддиалектной коммуникативной системы [7, с. 3]. Кроме того, литература XVI-XVII вв., а на этот период пришлись эпохи Возрождения и Реставрации, обладала ярко выраженным светским характером, ведущим жанром была драма [2, с. 159-161]; [3, с. 218-223]. Тексты пьес обладают особой ценностью при исследовании разговорной диалогической речи ушедших эпох, поскольку в репликах действующих лиц могут быть найдены фрагменты живой речи. Такими фрагментами, в частности, являются прагматические (дискурсивные) маркеры (слова, фразы, звуки, не имеющие реального лексического значения, но обладающие функцией формировать разговорную структуру, передавая намерения говорящих при разговоре [6]).

Формы и количество привлекаемых писателями-драматургами прагматических маркеров зависят от объективных и субъективных факторов. К объективным отнесём само существование на этапе написания произведения вербальных и невербальных средств создания фатических (неинформативных) элементов речи, то есть средств (с присущими им лексико-грамматическим составом и формами выражения), находящихся в речевом обиходе современников авторов (той аудитории, для которой и предназначаются произведения). К объективным факторам отнесём также продиктованную жанром произведения необходимость насыщения (в той или иной степени) реплик персонажей употребляемыми в повседневной речи привычными и узнаваемыми словами, конструкциями, звуками (как, например, современные нам средства русского языка - «наверное», «я думаю», «э-э-э», «хм-м»). Принятие автором решения о включении или не включении маркера в предложение, выбор разновидности маркера, определение его структуры (из предлагаемых повседневной речью языковых и речевых реалий, существующих на эта- 
пе написания произведения) и места его размещения в конструкции отнесём к субъективным факторам.

Ранее мы рассмотрели разнообразие прагматических маркеров в драматургических произведениях Ренессанса. В предложениях, отражающих прямую речь, нам встретились междометия tut, ha, ah, восклицания why, наречия then, surely, now, модально-предикативные сочетания I think, I presume, I know, I pray, обращения lady, sirs, woman, присоединенные вопросы и др. Говоря о размещении маркеров в предложении, мы отметили, что в том случае, если автор употребляет в предложении один маркер, последний размещается в начале, финале предложения или синтагмы, на границе клаузем в сложных структурах. Картина несколько меняется, если в предложении автор использует не один, а два или три маркера. Тогда композиции из двух или трёх маркеров, предикативных или непредикативных, состав которых варьирует, располагаются вокруг принимающего маркер предложения. Сочетания могут быть образованы союзом и наречием And surely, союзом и вводным предложением But I fear, междометием и обращением, как Tush, fool и Ah, sir, восклицанием и наречием Why, then и пр., такие сочетания образуют начальную часть предложения. Финальная часть формируется, например, присоединённым вопросом, или комбинацией нескольких (чаще двух) маркеров - обращения и присоединённого вопроса sir, are you?, наречия и вводного предложения now, I presume и др. Расположение фатических компонентов в виде комбинаций из двух или трёх маркеров внутри предложения - для выделения определённой группы слов, структурирования высказывания и других целей - встречается достаточно редко и представляет собой индивидуальный выбор создателя текста [11].

Мы также исследовали модально-предикативные сочетания, использованные одним из авторов Ренессанса, Николасом Юдаллом, в предложениях реплик пьесы «Ральф Ройстер Дойстер». Показано, что предикативные прагматические маркеры, которые употребляет автор, построены при помощи личного местоимения первого лица единственного числа и глаголом trust, pray, warrant, trot, wot, say, suppose, see, tell, advise, mean, confess, think. В целях придания естественности речи персонажей, Юдалл встраивает сочетания I think, I see, I trust и др. в повествовательные, императивные, вопросительные предложения. Маркеры выполняют в предложении и диалогическом тексте множество функций, среди которых в первую очередь следует назвать выделение определённых слов или словосочетаний, осуществление тема-рематического членения предложения, структурирование слишком объёмных конструкций устных реплик, выстраивание рифмы. Разнообразие рассмотренных вводных предложений формируется несколькими способами. В числе таких способов мы отметили употребление сокращённой и усечённой форм слов (сокращённой формы глагола advise и усечённой формы местоимения you - I'vise ye), распространение вводного предложения (дополнением I warrant - I warrant you; модальным глаголом I say - I dare say), изменение порядка слов при выдвижении глагола вперед (Mean I) [4].

Одной из употребляемых авторами Ренессанса структур является маркер I pray you, который в настоящем исследовании мы решили рассмотреть подробнее.

Считается, что вводный оборот I pray you, впервые засвидетельствованный в текстах конца XIV века в форме I pray thee, к XVI веку сократился до pray [5]. Однако полученные нами ранее результаты не позволяют согласиться с таким предположением. В этой связи представлялось интересным увеличить количество рассматриваемых текстов и на более обширном лингвистическом материале, расширив временные рамки исследования и обратившись к произведениям авторов не только периода Ренессанса, но и Реставрации, рассмотреть оборот I pray you / thee в синхронии, на этапах XVI и XVII вв.

\section{Материалы и методы исследования.}

Материалом для анализа послужили предложения из диалогов пьес, созданных английскими авторами в периоды Возрождения (1485-1660 гг.) и Реставрации (1660-1688 гг.). Всего были изучены тексты более 200 произведений. Для исследования применялись методы лингвистического исследования языковых фактов, проведения сплошной выборки изучаемого материала, сравни- 
тельно-сопоставительного анализа, лингвистического описания, трансформационная и оппозиционная методики. После иллюстрирующего примера в тексте будут указаны время написания или первой публикации произведения, его автор (если они известны), которые уточнялись по [8], [9], [12], [13], а также название и страница в источнике.

\section{Результаты и обсуждение}

Лексико-грамматические и стилистические характеристики рассматриваемых структур в художественном произведении, без сомнения, находятся во взаимосвязи и взаимозависимости, поэтому рассмотрим их в совокупности, уделив внимание следующим чертам оборота: порядок, число и формы слов, взаимодействие с другими маркерами, расположение в предложении.

Для проведения анализа примем за «обязательные» составляющие три слова: местоимениеподлежащее I, глагол-сказуемое pray и дополнение, выраженное местоимениями уои или theе и будем относиться к ним как к «изначально существовавшим» в конструкции оборота, тем более, что это не противоречит приведённым выше данным [5] и нашим наблюдениям за состоянием маркера в предложениях текстов XIV-XV вв.

Порядок «обязательных» слов в исследуемой структуре в нерифмованном тексте в основном един - «подлежащее + сказуемое + дополнение». Такую последовательность расположения составляющих видим в предложении I pray you answer me. (1575, Still J. “Gammer Gurton's Needle,” p. 235), где прагматический маркер оформляет императивное предложение. Отклонение от такого порядка расположения слов допускается автором в тех случаях, когда создаётся рифмованный текст и оборот располагается в конце строфы. Так, например, в диалогическом единстве -

"Neighbour Prat, come hither, I you pray -"

"Why, what is this nice fray?" (1533, Heywood J., "The Pardoner and the Frere", p. 23) -

императивное предложение реплики-зачина построено с привлечением двух прагматических маркеров - обращения Neighbour Prat и вводной клауземы I you pray. Употребление последней связано, скорее всего, с возможностью подобрать рифму для финального в реплике-реакции слова fray. Отсюда перемена мест рray и уои в обороте.

В следующем отрывке наблюдаем подобное явление - дополнение размещается между подлежащим и сказуемым с формированием вводной структуры I thee pray:

Merrygreek. Shall I tell him what ye say?

Custance. Yea, and add whatsoever thou

canst, I thee pray,

And I will avouch it, whatsoever it be. (1553, Udall N., "Ralph Roister Doister", p. 50).

Такой порядок слов продиктован репликой-зачином, произносимой Меригриком. Реплика показана автором в виде одной строфы, в конце которой употреблено слово say. В реплике-реакции оно рифмуется с pray, отсюда необходимость поставить местоимение theе перед глаголом.

Для первой половины рассматриваемого периода наиболее характерно включение в оборот всех трёх «обязательных» составляющих: I pray you, be content, and we will be more mannerly. (1568, Fulwell U., "Like Will to Like”, p. 322), I pray you, when did ye last kiss your cow? (1553, Udall N., "Ralph Roister Doister”, p. 23). Однако отмечаются и случаи сокращения количества слов. Редукции может быть подвержено дополнение, как в примере: I pray, good master, give me nine-pence. (1568, Fulwell U., "Like Will to Like", p. 349). Вводная структура с сохранённым дополнением, но опущением подлежащего, употребляется в предложении Pray you, speak English. (1614, Tomkis Th., "Albumazar”, p. 118).

Оба местоимения, формирующие как подлежащее, так и сказуемое, могут быть элиминированы. Предложение с вводной клауземой, представленной единственным словом, глаголомсказуемым pray, наблюдаем в примере Pray, is Monsieur Pisaro to de maison? (1598, Haughton W., “Englishmen for my Money”, p. 525). Такие формы обнаруживаются в текстах как XVI, так и XVII веков. Так, в реплике Pray how long is't, brother, since you left Spain? (1662, Tuke S., “The Adventures of Five Hours", p. 28) видим вводное предложение Pray, расположенное в начале конструкции и оформляющее вопросительное предложение. 
Стремления авторов к увеличению количества составляющих в обороте (относительно «обязательных») не наблюдается. Расширение группы подлежащего или сказуемого структуры I pray thee за счёт распространяющих слов не является характерным для изучаемого периода. Можно говорить, скорее, об индивидуальных предпочтениях или речевых привычках отдельных драматургов «усиливать» вводную структуру. Дополнительными элементами для использования в этих целях служат наречия, например, hartyly и now, размещённые в финале вводных клаузем, как в предложениях: But who shall do yt, I pray yow hartyly? (1538, Bale J., "Kynge Johan", p. 47) и I pray now, let me ask you one question that I remember. (1606, Day J., "The Return from Parnassus", p. 157). В следующем примере наблюдаем размещение между «обязательными» компонентами оборота, местоимением I и глаголом pray, ещё двух слов - наречия still и модального глагола shall: Send me such wenches, heavens, I still shall pray. (1598, Haughton W., "Englishmen for my Money", p. 486).

Отдельного внимания заслуживают формы слов, входящие в оборот. Так, в конце 1500-х гг. «в моду» входит совместное написание глагола pray и местоимения thee, причём структуры с местоимением-дополнением уои это не затрагивает. Можно предположить, что изменение написания глагола в этой разновидности оборота, в форме pry или pri, является следствием фонетических процессов, в частности, связано с редукцией гласного в слове pray. Другой тенденцией, приведшей к формированию такого правописания, может быть изменение логического ударения во фразе и, как следствие, сокращение паузы между словами pray и thee. В следующем примере видим вводную клаузему, в которой сохранены все три «обязательные» компонента, но показаны формой из двух слов, представляющих подлежащее $I$ и комплекс сказуемого и дополнения prythee: I prythee, note him well. (1602, Cooke J., "How a Man May Choose", p. 47). Несмотря на новый способ графического отражения и, скорее всего, произношения оборота, тенденция к сокращению составляющих затрагивает и его. Редуцированные структуры с элиминированным подлежащим, несколько отличающиеся друг от друга написанием, prithee и pr'ythee, обнаруживаются в конструкциях Prithee, hold thy peace. (1599, Shakespeare W., “The Merry Wives of Windsor”, p. 223) и And me too, pr'ythee. (1613, Jonson B., Fletcher Th., Middleton Th. “The Widow”, p. 234).

Далее обратимся к расположению клауземы I pray you / thee в конструкции. Если она является единственным прагматическим маркером в предложении, как в примерах: I pray you, come away to dinner. (1598, Greene R. "Mucedorus", p. 49) и Laugh at him, I pray you. (там же, р. 44), то наиболее характерно её расположение в начале или финале. Случаев вклинивания в информативную часть, независимо от длины предложения, нами не отмечено.

Если в предложении употреблены несколько прагматических маркеров, то возможны три основных варианта их расстановки.

Расположение в начале и финале конструкции можно видеть в примере Tush, lett me se them, I pray the hartely. (1538, Bale J., "Kynge Johan”, p. 35). Два маркера, восклицание tush,и вводная клаузема I pray the hartely как бы окружают императивное предложение lett me se them. В примере Why, hast thou spied the nee'le quickly, I pray thee tell? (1575, Still J. “Gammer Gurton's Needle," р. 232) видим подобное «окружение» у общевопросительного предложения. Конструкция начинается восклицанием why и заканчивается композицией из двух предикативных маркеров I pray thee и tell.

Все маркеры могут быть сосредоточены в начале предложения. В конструкции Why pray, how can you spend your time better? (1666, Killigrew W. "Pandora”, p. 4) наблюдаем редуцированную структуру pray, сопровождаемую восклицанием why, расположенную в начале предложения. Императив say not so в конструкции Nay, pray you, Master Lusam, say not so. (1602, Cooke J., "How a Man May Choose", p. 7) предваряется фатической частью, состоящей из восклицания Nay, вводной клауземы pray you и обращения Master Lusam. В начале предложения I praye the hartely tell me why thu doest so? (1538, Bale J., “Кynge Johan”, p. 98) наблюдаем сосредоточение вводного I praye the hartely и императива tell me. Последовательность из союза and, вводной клауземы I pray thee и императива tell me видим в начале вопросительного предложения And I pray thee tell me, is not thy name New Custom? (1573, Daiches S., "New Custom”, p. 22).

Все прагматические маркеры могут быть размещены в финальной части предложения. Таково, например, расположение обращения sir и вводного I pray you heartily в структуре Stay yourself, sir, 
I pray you heartily. (1568, Fulwell U., "Like Will to Like", p. 322). В конструкции This assurance how cometh it, declare, I pray you? (1573, Daiches S., "New Custom", p. 46) наблюдаем сосредоточение двух предикативных маркеров, declare и I pray you, в постпозиции от вопросительного предложения.

В том случае, если принимающее маркеры предложение является сложным, тенденция к расположению маркеров не меняется. Они группируются на границе клаузем, то есть в начале одной клауземы и /или в финале другой. В конструкции May not folks be honest, pray you, though they be poor? (1553, Udall N., "Ralph Roister Doister", p. 60) наблюдаем два маркера, вводное предложение pray you и союзное наречие though, которое, согласно классификации Б. Фрейзера, может быть отнесено к контрастивным маркерам [10]. В предложении Yea, and add whatsoever thou canst, I thee pray, and I will avouch it, whatsoever it be. (1553, Udall N., "Ralph Roister Doister", p. 50) маркеры располагаются в начале конструкции и на границе клаузем. Подобное же расположение вводного предложения и обращения наблюдаем в примере Pray how long is't, brother, since you left Spain? (1662, Tuke S., “The Adventures of Five Hours", p. 28).

Можно предположить, что функция разновидностей структуры I pray you/thee в живой речи изучаемого периода, не зависимо от того, находится ли она в составе фатического «блока» или же является единственным прагматическим маркером в конструкции, связана с местом размещения. При расположении в начале конструкции основным её назначением служит привлечение внимания собеседника, при расположении в концовке - смягчение категоричности высказывания. Эмотивная составляющая также присутствует - употреблением маркера адресант демонстрирует настойчивость в желании получить ответную реакцию адресата, акциональную или вербальную. В тексте пьесы функции I pray you/thee, несомненно, другие. Авторы включают маркер в предложения реплик персонажей, используя те его формы, которые употребляются современниками в повседневной жизни, для придания как можно большей естественности речи действующих лиц. Размещая маркер в объёмных конструкциях, состоящих из нескольких клаузем, его используют как средство, структурирующее отрезок речи актёра для облегчения восприятия слушающим (зрителем), как средство заполнения паузы. При создании рифмованного текста авторы располагают маркер в конце строфы, при этом возможности для подбора рифмы расширяются, благодаря неустойчивому порядку слов и непостоянному количеству составляющих в изучаемой структуре.

Рассматривая вводное предложение I pray you / thee в диахронии, можно заключить, что форма представления «обязательных» составляющих оборота в XIV-XV вв. отличается большим разнообразием. Так, глагол записывается в виде pray или praye c корневой $a$, и, кроме того, следуя старофранцузскому слову preier, с корневой $e$ - preye или prey. B XVI в. идёт процесс постепенного приведения к единообразию написания глагола и нам встретились только формы praye и pray. Также намечается тенденция к стандартизации написания местоимения, служащего дополнением, и из разнообразия форм местоимений 2 лица, которые в обороте XIV-XV вв. выглядят как yow или thee, а также the, pe, остаются две - уои и thee.

Количество «обязательных» слов в обороте с течением времени уменьшается. Если для XIV$\mathrm{XV}$ вв. характерно сохранение всех трёх составляющих и, кроме того, в структуру могут включаться и дополнительные элементы (наречия, модальные глаголы), то в первой половине XVI в. такой подход авторов к отражению оборота в тексте сохраняется, а во второй половине XVI и в XVII вв. наблюдается тенденция к элиминированию «обязательных» местоимений, подлежащего и дополнения, с образованием структуры pray. Таким образом, можно говорить о характерном сокращении оборота I pray you / thee до pray, скореe, не к XVI в., а к концу XVII века.

Словорасположение во вводном предложении I pray yow / thee в XIV-XV вв. не имеет определённых норм. Авторы успешно используют способность оборота менять порядок составляющих и размещают его в конце строфы, обеспечивая возможность рифмования с нужным словом. Так, в примере

\section{Dere leman, $\boldsymbol{y}$ prey $\mathbf{p e}$}

Pi wille Patow tel to me. (1300s, "The Romance of Guy Warwick")

слово Ре поставлено в обороте в постпозицию от глагола с целью подобрать рифму для завершающего следующую строфу слова те. Иной порядок компонентов во вводном предло- 
жении, с расположением глагола-сказуемого после местоимения-дополнения, можно видеть в отрывке

Isaac: when I am dede, and closed in clay,

who shall then be youre son?

Abraham: A, lord, that I shuld abide this day!

Isaac: sir, who shall do that I was won?

Abraham: speke no sich wordis, son, I the pray. (1300s, "Abraham. The Towneley Plays").

В нём поэтический текст имеет сложную структуру, первая, третья и пятая строфы рифмуются между собой. Первая и третья заканчиваются словами clay, day. Финальная клаузема в предложении пятой строфы должна заканчиваться словом pray, поэтому сказуемое и дополнение во вводном предложении меняются местами. Таким образом, порядок составляющих в обороте обусловлен запросами рифмы и может легко перестраиваться.

Авторы Ренессанса также применяют приём перемены мест сказуемого и дополнения для рифмованного текста. Если оборот употребляется в предложении нерифмованного текста, в нём устанавливается порядок слов «подлежащее + сказуемое + дополнение».

Оформление вводного оборота в виде главной клауземы, как это наблюдается, например, в «Кентерберийских рассказах» I pray yow alle that ye nat yow greve; (1380-1390-e, Chaucer G., “The Reves Tale"), в XVI-XVII вв. уже не встречается.

Если в предложении XIV-XV вв. сочетание I pray you / thee является единственным прагматическим маркером, оно размещается преимущественно в начале или финале конструкции. Такая тенденция сохраняется и в последующие два века. Если же в предложении употребляется несколько маркеров, то они сконцентрированы также либо в начале, либо в финале, то есть «точечно». Кроме того, наблюдаются варианты «рамочного» размещения от двух до пяти маркеров в конструкции, когда в пре- и постпозиции от принимающего предложения находятся не только I pray yow / thee, но и непредикативные маркеры, например, обращения (gode suster, dame, son, sir, good sir,), союзы (and), наречия (therforee, nowe, now, specially, yet), устоявшиеся сочетания (for goddess sake), восклицания (loo), а также предикативные, как telleth me, tell me. В произведениях XVI в. разнообразие сочетаемых с оборотом маркеров несколько уменьшается, уменьшается также и количество маркеров, включаемых в одно предложение вместе с I pray yow / thee. К концу XVII в. вместе с I pray / pray в предложении размещаются в основном восклицания (why), союзы (and), обращения (sir).

\section{Заключение.}

В работе исследованы предложения, содержащие вводную клаузему I pray you и её разновидности. Лексический материал подбирался из текстов диалогов пьес, созданных в XVI и XVII вв.

Показано, что начало рассматриваемого периода характеризуется наибольшим разнообразием форм изучаемого оборота. Это разнообразие формируется из-за нескольких процессов, действующих на вводное предложение. Первый - увеличение собственного объёма клауземы путём добавления наречий (как now, still, hartyly), модальных глаголов (например, shall). Второй - изменение в оформлении и, вполне вероятно, произношении составляющих слов с появлением разновидности I prythee и её вариантов. Третий процесс - редукция компонентов. Элиминированию подвержены местоимения - подлежащее и / или дополнение. Ближе к концу рассматриваемого периода наблюдается некоторое уменьшение разнообразия и сужение количества разновидностей преимущественно до форм I pray, pray, prythee.

Располагая оборот относительно принимающего предложения, авторы дополняют его другими маркерами, предикативными (как tell me, tell, declare) и непредикативными (but, why и др.). Сочетания нескольких прагматических маркеров размещаются преимущественно в начале или финале конструкции или входящих в её состав клаузем, что наблюдалось также и в текстах XIV-XV вв.

(C) Меркурьева Н.Ю., 2021 


\section{Источники иллюстративного материала}

Bale John. Kynge Johan. A play in two parts. By John Bale. London: Ed. J. Bowyer Nichols and Son, 1838. 110 p.

Cooke Joshua. How a Man May Choose a Good Wife from a Bad. A Select Collection of Old English Plays. V. 9. London: Reeves and Turner, $1874576 \mathrm{p}$.

Daiches Samuel. New Custom. A Select Collection of Old English Plays. V. 3. London: Reeves and Turner, 1874. 359 p.

Day John. The Return from Parnassus: or, the Scourge of Simony. A Select Collection of Old English Plays. V. 9. London: Reeves and Turner, 1874. $576 \mathrm{p}$.

Fulwell Ulpian. Like Will to Like. A Select Collection of Old English Plays. V. 3. London: Reeves and Turner, 1874. 359 p.

Greene Robert. The Comedy of Mucedorus. Halle: Ed. Max Niemeyer, 1878. 79 p.

Haughton William. Englishmen for my Money; or, A Woman Will Have Her Will. A Select Collection of Old English Plays. V. 10. London: Reeves and Turner, 1874. 564 p.

Heywood John. The Mery Play between the Pardoner and the Frere, the Curate and Neybour Pratte. The Dramatic Writings of John Heywood. Early English Dramatists. Vol. 5. London: Ed. John S. Farmer, 1905. 280 p.

Jonson B, Fletcher Th., Middleton Th. The Widow. A Select Collection of Old Plays. V. 12. London: Ed. Isaak Reed, 1827. $422 \mathrm{p}$.

Killigrew William. Pandora: a Comedy or the Converts. Fovr New Playes written by Sr. William Killigrew. Oxford: Ed. Ric. Davis, 1666. 246 p.

Shakespeare William. The Merry Wives of Windsor. The Works of William Shakespeare in nine volumes. V. 1. Cambridge and London: Macmillan \& Co, 1863. P. 161-292.

Still John. Gammer Gurton's Needle. A Select Collection of Old English Plays. V. 3. London: Reeves and Turner, 1874. 359 p. Tomkis Thomas. Albumazar. A Select Collection of Old Plays. V. 7. London: Ed. Isaak Reed, 1825. 456 p.

Tuke Samuel. The Adventures of Five Hours. A Tragi-comedy. A Select Collection of Old Plays. V. 12. London: Ed. Isaak Reed, 1827. $422 \mathrm{p}$.

Udall Nicholas. Ralph Roister Doister. The Dramatic Writings of Nicholas Udall. London: Ed. John S. Farmer, 1907. 160 p.

\section{Список литературы}

1. Аракин В. Д. История английского языка. М.: Физматлит, 2009. 304 с.

2. История английской литературы. Т.1, вып. 1. М-Л: Изд-во АН СССР, 1943. 324 с.

3. История английской литературы. Т.1, вып. 2. М-Л: Изд-во АН СССР, 1945. 655 с.

4. Меркурьева Н.Ю. Предикативные прагматические маркеры в предложениях текста пьесы XVI в. // Гуманитарные и социальные науки. 2020. № 4. С. 138-148.

5. Online Etymology Dictionary. URL: https://www.etymonline.com (дата обращения 10.03.2021)

6. Словарь лингвистических терминов Жеребило. URL: https://gufo.me/dict/linguistics_zherebilo (дата обращения 19.09.2021)

7. Швейцер А.Д. Американский вариант литературного английского языка: пути формирования и современный статус. // Вопросы языкознания. 1995 № 6. С. 3-16.

8. The Dictionary of Literary Biography. V. 18, 21, 32, 39, 58, 62. Michigan: A Briccoli Clark Book.1982-1987.

9. A Dictionary of Literature in the English Language. V. 1-2. Oxford: Pergamon Press, 1970.

10. Fraser B. What are discourse markers? // Journal of Pragmatics. 1999. № 31. P. 931-952.

11. Merkuryeva N.Yu. Pragmatic markers in the sentences from Renaissance artworks. Сборник материалов международной научной конференции «Наука. Образование. Практика» Ч. 2. Торонто: Инфинити, 2020. С. 79-84.

12. The New Cambridge Bibliography of English Literature. V. 1-5. Cambridge: At the University Press, 1977.

13. Reference Guide to English Literature. V. 1-3. Chicago and London: St. James Press, 1991.

\section{References}

1. Arakin, V.D. Istoriia angliiskogo iazyka [A history of the English language] / V.D. Arakin. Moscow: Fizmatlit, 2009. 304 p.

2. Istoriia angliiskoi literatury [The History of the English Literature]. Vol. 1. Issue 1. Moscow-Leningrad, Academy of Science Publ. 1943. 324 p.

3. Istoriia angliiskoi literatury [The History of the English Literature]. Vol. 1. Issue 2. Moscow-Leningrad, Academy of Science Publ. 1945. 655 p.

4. Merkuryeva, Natalia Y. Predicativnye pragmaticheskie markery v tekste piesy XVI v. [Predicative pragmatic markers in the XVI century play text sentences] / N.Y. Merkuryeva // Gumanitarnye i sotsialnye nauki [Social and Human Sciences Journal]. 2020. № 4. P. 138-148.

5. Online Etymology Dictionary, https://www.etymonline.com.

6. Slovar lingvisticheskih terminov Zherebilo. [Zherebilo Dictionary of linguistic terms], https://gufo.me/dict/linguistics_zherebilo. (дата обращения 19.09.2021)

7. Shveitser, A.D. Amerikanskii variant literaturnogo angliiskogo iazyka: puti formirovaniia i sovremennyi status./ A.D. Shveitser // Voprosy Iazykoznaniia [Voprosy Jazykoznanija (Topics in the Study of Language)]. 1995. № 6. P. 3-16.

8. The Dictionary of Literary Biography. V. 18, 21, 32, 39, 58, 62. Michigan: A Briccoli Clark Book, 1982-1987. 
9. A Dictionary of Literature in the English Language. V. 1-2. Oxford: Pergamon Press, 1970.

10. Fraser, B. What are discourse markers? // Journal of Pragmatics. 1999. № 31. P. 931-952.

11. Merkuryeva, N.Yu. Pragmatic markers in the sentences from Renaissance artworks. Materials of the International Scholarly Conference «Science. Education. Practice». Part 2. Toronto: Infinity, 2020. P. 79-84.

12. The New Cambridge Bibliography of English Literature. V. 1-5. Cambridge: At the University Press, 1977.

13. Reference Guide to English Literature. V. 1-3. Chicago and London: St. James Press, 1991.

\section{Сведения об авторе:}

Меркурьева Наталья Юрьевна - кандидат химических наук, доцент кафедры юриспруденции МГУУ ПМ им. Ю.М. Лужкова. Область научных и профессиональных интересов: теоретическая грамматика, грамматика разговорной диалогической речи, исторический синтаксис английского языка.

E-mailnata2lya1@mail.ru

\section{About the author:}

Merkuryeva Natalia - PhD, Associate Professor of Moscow Metropolitan Governance Yury Luzhkov University (Moscow, Russia). Spheres of research interest: theoretical grammar, spoken language grammar, historical syntax. E-mail: nata2lya1@mail.ru 